Published in 2007

'The Idea of Contrastive Explanandum', in Rethinking Explanation, edited by J. Persson and P. Ylikoski, Boston Studies in the Philosophy of Science 252, Springer: 27-42.

\title{
The Idea of Contrastive Explanandum
}

\author{
Petri Ylikoski \\ Helsinki Collegium for Advanced Studies \\ University of Helsinki \\ Petri.ylikoski@helsinki.fi
}

In this paper, I will discuss the idea of contrastive explanandum. I will restrict my discussion to singular causal explanation, but the basic ideas and the arguments have a broader application. They are relevant also to other kinds of explanations. In the first section I will first present the intuitive idea of contrastive questions, and then elaborate it by discussing typical criteria for the choice of a contrast. I also suggest a novel way to see the difference between scientific and everyday explanatory questions. In the second section I will discuss the major criticisms presented against contrastive theories of explanation in order to further clarify my position. I argue that all explananda can be analyzed as contrastive and that this is a fruitful approach in understanding explanatory questions. I also argue that the contrastive thesis should be understood as a claim about what an explanation can explain, not as a thesis about what the explainee has in her mind. Finally, I defend the thesis that a contrastive explanandum can be reduced to a non-contrastive explanandum against the arguments presented by Dennis Temple and John W. Carroll.

\section{The contrastive explanandum}

A famous joke about the bank robber Willie Sutton introduces the basic idea of contrastive explanation. When Willie was in prison, a prison priest asked him why he robbed banks. Willie answered, "Well, that's where the money is." The joke is based on a confusion, for Willie was not answering the question the priest was asking. The priest had in his mind the question: "why do you rob banks, instead of leading an honest life?", whereas Willie answered the question: "why do you rob banks, rather than gas stations or grocery stores?" This is the basic insight of the contrastive approach. We do not explain simply 'Why $f$ ?' rather, our explanations are answers to the contrastive question 'Why $f$ rather than $c$ ?'. (Garfinkel 1981: 21-22.) Instead of explaining plain facts, we are explaining contrastive facts. Several philosophers of explanation have used the same basic idea. (For example, Hart \& Honoré 1959; Hansson 1975; van Fraassen 1980; Garfinkel 1981; Hesslow 1983; Woodward 
1993 (originally published in 1984); Lewis 1986; Sober 1994 (originally published in 1986); Temple 1988; Lipton 1990, 1991, 1993; Barnes 1994; Hitchcock 1996, 1999; Carroll 1997, 1999; Risjord 2000.) I will follow their lead and try to develop the contrastive idea a little bit further.

In the following discussion I am not committed to any specific theory about explanationseeking questions. (See van Fraassen 1980; Tuomela 1980; Sintonen 1984; Koura 1988; Hintikka \& Halonen 1995.) Some advocates of the contrastive approach subscribe to the thesis that all explanatory questions are always why-questions (van Fraassen 1980). The account presented here does not include any such commitment. The thesis that explanations are answers to questions should be kept separate from the thesis that all explanation-seeking questions are why-questions. As I see it, the same explanatory request can often be made using various linguistic devices (Scriven 1959: 451). For example, in some cases a howquestion is a more natural way of making a contrastive explanatory request than a whyquestion. From the point of view of my account, it is not essential whether every explanationseeking question is a why-question or that all explanation-seeking questions can be paraphrased as why-questions. As Markwick (1999: 191) has noted, there is no deep commitment to any specific question-theoretical approach among most supporters of the contrastive theory of explanation. I will continue this tradition of non-commitment.

Contrary to a common misunderstanding, the erotetic approach to explanation is fully compatible with the realist account of explanation. It is not confined to the pragmatics of giving explanations nor does it commit one to the kind of explanatory subjectivism advocated by Bas van Fraassen (1980). As I have argued earlier (Ylikoski 2001), the contrastive account is completely compatible with view that the aim of explanations is to track objective relations of dependency in the world. Explanations are about the things in the world and there is more to the explanation than that the recipient is satisfied with it, as the extreme pragmatic theory of explanation would have it. It is possible that the recipient is wrong in accepting a certain answer as an explanation. Although the question is wholly up to the recipient, the adequacy of the answer is not.

In fact, the idea that explanations are contrastive is natural if one thinks that the aim of explanation is to trace relations of dependency and that questions are crucial element in understanding explanation. In this context the idea that explanations are answers to "what-ifthings-had-been-different' questions (Woodward 1993, 2003) is quite natural. We wish to know how the change in the causes brings about the change in the effects. We want to know what makes the difference and then to leave out factors that do not. Furthermore, if there is no 
change in the effects when we make changes to our putative explanatory factors, then we do not have truly explanatory factors, since there is no appropriate relation of dependency.

The contrastive idea works nicely with our preferred form of causal inquiry: the scientific experiment. When we are looking for explanations using the methods of experimental inquiry, we are basically working in a contrastive setting. For example, we contrast the control group with the experimental group or the process after the intervention with the process before the intervention. In both of these cases, we are trying to account for the differences between the outcomes. The basic idea is to keep the causal background constant and bring about changes in the outcomes by carefully controlled interventions. The same contrastive setting also works in comparative research, which is our second option if experiments are impossible. Clearly, by adopting the contrastive idea, we are starting with a very intuitive and central feature of our cognition. (Hilton 1995)

It can be speculated that our contrastive explanatory preferences stem from our nature as active interveners in natural, psychological, and social processes. We want to know where to intervene to produce the changes we want, and this knowledge often presupposes answers to some why- and how-questions. Without this knowledge we would not know when the circumstances are suitable for our intervention. We would not be able to predict the results of our interventions. I am not claiming that we can reduce the notion of explanation to its origins in agency. We also want explanations for things that we cannot manipulate. However, our instrumental orientation might still explain why our explanatory preferences are as they are. Causal explanations face the problem of explanatory selection (Hesslow 1983). We have to pick the right aspects of the causal process to be included in the explanation. All causal information is not explanatory information. Usually, the causal history of an event includes a vast number of elements and aspects that are not explanatorily relevant to the explanationseeking question we are addressing. We want only the items that make a difference in the things that we are interested in. But the problem of explanatory selection is wider than that, for it also requires that the explanans must also be described in the right way. Things can be described in various ways and at various levels of abstraction, and the challenge is to find the right way and the right level for the explanation at hand. The crucial advantage of the contrastive approach is that it allows us to be specific about the explanandum. As a consequence, it can be profitably used in the analysis of apparently competing explanations. As it will turn out, many apparently competing explanations are in reality complementing explanations. 


\section{Varieties of contrastive explanation}

There are various sorts of contrastive explanation, depending on the nature of the explanandum. They all share two basic ideas of the contrastive explanation. In all of them, the explanation traces objective relations of dependence and it is seen as an answer to a contrastive question. These explanations differ in terms of their explananda and in terms of the kind of dependence relationship they are tracking.

First, there is singular causal explanation, which is the topic of this paper. In singular causal explanation we are explaining facts about events in terms of facts about the earlier development of the causal process in question. For example, we explain facts about a car accident by referring to the facts about events and circumstances that occurred before the accident. The explanation aims to select the relevant facts from the causal history, the measure of relevance being their contribution to the difference between the fact and its foil. I will return to this explanatory setting shortly.

Second, there is a singular explanation for an instantiation of a property. For example, we might explain the fragility of a glass by referring to some facts about its molecular structure. This explanation does not directly refer to causal processes, or causal dependency, between molecular structure and fragility, but to the relations of dependency between the properties. The fact that a glass has a certain molecular structure constitutes the fact that it is fragile. Certain facts about the molecular structure make the difference between being fragile and being something else. They also determine the specific way in which the glass is fragile. As all facts about the molecular structure of the glass are not relevant to its fragility, we have a similar problem of explanatory selection as in the case of singular causal explanation. Furthermore, as there might be, and probably are many different ways to constitute fragility, our singular explanation of property instantiation is not necessarily a general explanation of the property of fragility. (For an account of this kind of explanation, see Cummins 1983.)

Regularities and laws are the third important class of explananda where the contrastive model of explanation is applicable. In such cases we are interested in the dependence between regularities and more fundamental laws and mechanisms. The laws or regularities to be explained are the way they are because the more fundamental laws and mechanisms are the way they are. The contrastive approach also works here: when explaining laws and regularities, we are explaining why they are the way they are, rather than otherwise. This short survey of various kinds of explanation is not intended to be exhaustive. The central point here is that the intuitions behind the contrastive approach are general intuitions about 
explanations, and not ad hoc specifications made to suit the case of singular causal explanation.

\section{Indicating the contrast}

There are various linguistic means to indicate the contrast in an explanation-seeking question (van Fraassen 1980: 128; Garfinkel 1981: 25, 29; Sober 1994: 176). For example, in English we can ask:

Why $f$ rather than $c$ ?

Why $f$ and not $c$ ?

Why $f$ instead of $c$ ?

Given that $f$ or $c$, why $f$ ?

The contrast can also be indicated by the combination of emphasis and non-linguistic contextual cues. Sometimes the contrast is so obvious from the context that there is no need to indicate it at all. The existence of alternative linguistic means even within a single language suggests that there is no single privileged way of indicating the contrast. (contra Carroll 1997, 1999.)

In order to simplify the presentation and to avoid possible problems of generalizing the curiosities of one particular linguistic way of indicating the contrast, I will denote the contrastive explanandum by $f[c]$. Here $f$ is a fact, and $c$ is a non-compatible alternative (a foil) to $f$. This notation allows one to state the intended explanandum more clearly, or at least more economically. The usual linguistic devices can be ambiguous and clumsy in complicated situations that arise in philosophical discussion. The use of technical notation also underlines the difference between understanding the structure of the explanandum on one hand, and the pragmatics of locutions like '... rather than ...' in English language on the other hand.

Some of the pioneers of the contrastive approach (for example, Bas van Fraassen and Alan Garfinkel) speak about a contrast class instead of a single contrast. I think this way of speaking is misleading from the point of view of analyzing explanation. The basic unit of explanation is always an explanation of singular $f[c]$. The contrast class $f\left[c, c^{*}, c^{* *}\right.$, etc. $]$ can always be understood as cluster of more simple explananda and be partitioned to more simple explananda: $f[c], f\left[c^{*}\right], f\left[c^{* *}\right]$, etc. This observation does not rule out that in practice a given piece of explanatory information can explain more than one contrast. In such case its just contains more than one explanation. A single answer can sometimes answer more than one question. It is also possible that a whole group of contrasts can be equivalent from the point of view of explanation. In this case the same explanatory information explains are explananda $f$ 
$[c], f\left[c^{*}\right], f\left[c^{* *}\right]$. Case like this can be found for example in equilibrium explanations. However, neither the excess of explanatory information nor the explanatory equivalence of some contrasts is an argument against the idea that the basic unit of explanation is the singular contrastive fact.

\section{The ideal explanatory text}

There are two basic approaches to identify the basic unit of explanation that are often seen as incompatible. The issue is about the standard for the completeness of explanation. The erotetic approach advocated in this paper regards an answer to an explanation-seeking (contrastive) question as a basic unit of explanation. For the advocates of the other view this is just an observation about pragmatics of requesting and giving explanations. They separate the answers and explanation proper by saying that the actual explanations by people just provide information about the real, and more complete, explanation. Peter Railton (1978, 1981; see also Hållsten 2001) calls this more inclusive unit of explanation an ideal explanatory text.

According to Railton, the basic goal of science is the subsumption of particular facts and regularities to the 'nomic nexus'. This is achieved by fitting the world's phenomena into a fully general and comprehensive theory. In Railton's vision, the ideal for which science strives is the 'ideal explanatory text', which would be able to explain every aspect of the phenomenon under consideration and would that also have a deductive-nomological structure. He notes, that "... plainly there is no question of ever setting such an ideal text on paper" (Railton 1981: 247), but he wants to underline the regulative role of such ideal. The aim of science is to develop a capacity to provide material for such ideal explanatory texts. I do not want discuss the merits of Railton's ideal as a description of the goal of scientific knowledge. He clearly describes a picture of science that is accepted by many philosophers of science, but the validity of this picture is not an issue here. The question concerning the ideal form of scientific knowledge is a separate issue from the question 'what makes a given explanation explanatory?'.

The trouble with the idea of the ideal explanatory text is that it does not give us any hints about the principles that govern the choice of explanatory information. Real life explanations are said to provide information about the ideal explanatory text, but Railton himself admits that he has no conceptual tools to cash out this idea. (Railton 1981, 244; Woodward 2003, 175-181) Obviously, his approach requires additional ideas to handle this central problem. In practice, only the erotetic approach can do this. As a consequence, in order to have full 
account of explanation, the advocate of the ideal text approach needs both ideas. In this sense the two approaches are not real alternatives. The supporter of ideal text approach needs both. The situation is different from the point of view of erotetic approach. Its supporters can claim that they can have all the advantages of the ideal text approach without accepting any of its philosophical presuppositions. At least, this is something that I would like to argue. The basis of my argument is the possibility that the concepts of complete explanatory text and ideal explanatory text can be characterized using the idea of contrastive explanandum. A complete explanatory text for the fact $f$ would contain all information required for answering any possible contrastive question $f[x]$ about the $f$. The construction of the complete explanatory text would involve explaining why $f$ against all possible contrasts. The concept of the ideal explanatory text would be even more ambitious. Here we would have a combination of complete explanations for any fact $f$ about some event $e$. The ideal explanatory text would literally explain everything about $e$.

With the help of these concepts, the advocate of the erotetic approach can claim that she can, if she wishes, say that the ideal aim of science is to provide ideal (or complete) explanatory texts as Railton has suggested. She can also claim that the erotetic approach is more fundamental. The elements of the ideal explanatory text for the event $e$ are explanatory because they are parts of adequate answers to some contrastive questions about $e$. However, there is no way in which the supporters of the ideal text approach can accomplish a similar derivation. This asymmetry between the two approaches suggests that the erotetic approach is conceptually more fundamental.

\section{Explanatory adequacy and failure}

We have an adequate explanation of $f[c]$ when we have explained why $f$ happened rather than the intended contrast $c$. An explanation is inadequate when it does not explain $f[c]$. An explanation can fail in various ways (Lewis 1986: 226-228). We can distinguish between a broad and narrow sense of explanatory failure. An explanation fails in the narrow sense due the fact that the offered explanation does not fulfill the requirements of an adequate explanation (see Ylikoski 2001: Chapter 2). For example, an explanation can fail by being partial. In such a case, the provided information is explanatorily relevant and true, but the explanation needs to be supplemented to be fully adequate. This notion is especially important in the case of probabilistic explanation. (Ylikoski 2001: Chapter 3) However, its use is not limited to probabilistic contexts. 
In the broad sense an explanation can fail in two different ways. First, the explanation might provide misinformation. It can claim things that are not true. Let us call this a factual failure. Although in practice it can often be very difficult to determine the facts of the matter, the case of misinformation is not a big theoretical problem for the theory of explanation. A distinction between a possible explanation and the true explanation can be useful for avoiding confusion. A possible explanation satisfies all the other criteria of a good explanation except for the truth requirement. If it were true, it would explain the explanandum. It fails for purely factual reasons. Another kind of factual failure is the failure to correct incorrect presuppositions of the explanatory question. In such cases the explainer fails to point out to the recipient that her question rests on premises that are not true. This failure can also be classified as an example of pragmatic fallacy, but I think it is clearer to treat it as an example of factual failure.

The second possible explanatory failure in the broad sense is pragmatic failure. In these cases the explainer does not provide what the recipient of the explanation wants. For some reason, there is a communication breakdown between the explainer and the recipient. These failures are similar in all forms of communication, and consequently, they are not unique to the communication of explanatory information.

Again, a pragmatic failure can occur in a number of ways. First, the explanation can answer the wrong question. The explanation could be perfectly good, but it does not address the question that the recipient wants to be answered. It might be that the recipient already knows that answer or she simply does not care about that particular question. Second, the explanatory information might be in a form that the recipient cannot understand. The explanation might be so technical, or the vocabulary so full of jargon, that the recipient cannot cope with it. The explainer might also presuppose background knowledge that the recipient does not have, which leads to a failure to understand. The third way to fail pragmatically is to provide the explanatory information in such a form that the recipient cannot separate the explanatory information from all the other information provided. In such cases, the explainer provides the explanatory information and so much other information that the recipient cannot disentangle them. A similar failure happens when I ask for Frank's telephone number, and someone provides me all the numbers in the telephone book.

\section{How do contrasts arise?}

Eric Barnes (1994: 37) warns about taking a linguistic approach to the generation of contrasts. He notes that most writers on contrastive explanation have used substitutional transformation of sentences to generate the contrasts. 
Halonen won the 2000 Finnish presidential election

[Aho won the 2000 Finnish presidential election,

Hautala won the 2000 Finnish presidential election, ...]

This way of presenting contrasts is obviously both pedagogically and stylistically practical, but it can give a misleading impression that this is the right or the only way to generate contrasts. For example, the following two suggestions are sensible contrasts, but they cannot be generated by substitutional transformation:

Halonen won the 2000 Finnish presidential election

[The 2000 Finnish presidential election ended in a tie]

or

Halonen won the 2000 Finnish presidential election

[The results of the 2000 Finnish presidential elections were nullified]

Clearly, our focus should be on the contrasted states of affairs, not on their linguistic representations. But how do we arrive at these contrasting states of affairs? There is more than one way to come up with a contrast. It can arise either by imagination or by comparison, or by a combination of the two.

In everyday life, explanatory questions most often arise as a consequence of an abnormal or unexpected incident. Something abnormal happens and raises our curiosity. We want to know why it happened. In such situations the choice of the contrast is obvious: we will contrast the abnormal occurrence with the normal or expected state of affairs. (Hart \& Honoré 1959: 3138.) In his important 1983 paper, Germund Hesslow distinguishes five different ways in which the contrast can arise. It is useful to summarize these cases, since they illustrate various senses of 'normality' in explanatory context.

First, the contrast can be the statistically normal case. For example, when we ask why a particular barn caught fire, we are asking what distinguishes the barn under consideration from other barns. And when we consider these other barns, which are made of similar materials, placed similarly and used similarly, we find that most barns of this type have not burned. This fact is our contrast. We will be looking for causal factors that are present in the case of our burned barn, but not in the statistically normal case. (Hesslow 1983: 95.)

Second, the contrast can be the temporally normal case. When we ask why the barn caught fire at some particular time, we are comparing the time of the fire with the barn at earlier times. So here we are not comparing different but similar objects, but the same object at different times. In this case we will be looking for some changes in the conditions to account for the change in the states of affairs we are interested in. (Hesslow 1983: 95.) 
The third possible contrast is a theoretical ideal. Here the contrast does not arise from the observation of a difference, but from the predictions or assumptions of our theory. A theoretical account gives us a kind of 'default' contrast. The use of this kind of contrast facilitates the systematization of the field covered by the theory. Hesslow mentions Max Weber's 'ideal types', the equilibrium models of the perfect market in the neo-classical economics, the definition of a 'wild type' in genetics, and the physiology of the healthy organism in medicine as typical examples of such theoretical ideals. (Hesslow 1983: 95-96.) These all provide scientists with a standard of comparison that helps in picking the things to be explained. Hesslow also compares theoretical ideals with what Stephen Toulmin calls ideals of natural order. Toulmin writes:

Our 'ideals of natural order' mark off for us those happenings in the world around us which do require explanation, by contrasting them with 'the natural course of events' - i.e. those events which do not. Our definition of the 'natural course of events' is therefore given in negative terms: positive complications produce positive effects, and are invoked to account for deviations from the natural ideal, rather than conformity to it (Toulmin 1961: 79).

Since both Hesslow and Toulmin are very brief in characterizing them, it is difficult to say whether their concepts are the same, but at least one can say that their function in the context of explanation seems to be the same. Both work as generators of contrasts for scientific explanatory questions.

The fourth source of a contrast is subjectively expected. Here the contrast is what the agent was expecting to happen. These explanations show how the fact to be explained could have occurred against the expectations we had on the basis of knowledge of earlier conditions. (Hesslow 1983: 96.) This source of contrast is interesting because it can be related to the intuition behind the original covering-law theory. According to Hempel, the function of the explanation is to make the explanandum expected. (Hempel 1965: 337.) He later interpreted this intuition as a requirement that the explanation must make the explanandum highly probable. The contrastive analysis gives an alternative way of interpreting this intuition: many explanations are related to our expectations since we are typically explaining facts that do not accord with our expectations. Showing why the unexpected happened corrects our background beliefs and in this sense reduces the unexpectedness of the explanandum. If this is right, we can give up the requirement that the explanation must make the explanandum highly probable, without losing the intuition that, at least sometimes, the function of an explanation is to reduce surprise. 
The fifth possible source of a contrast is a moral ideal. Sometimes an action is contrasted with a normative account of how an agent should have acted. In such a case we are asking for the explanation for the deviance from this standard of conduct, and we choose as explanatory causes such conditions that should not have been present. (Hesslow 1983: 96-97.)

This is not an exhaustive list of all possible ways in which a contrast can arise. Certainly there are also other ways of generating contrasts. It is essential to see that there are various ways in which a contrast can arise, but having an exhaustive list of all the ways they can arise is not. However, there is one way in which Hesslow's list can be misleading. All the explananda in his list are either abnormal or unexpected. This goes nicely with our everyday practices of explanation. However, we sometimes also want to explain the normal case. For example, we might want to explain why grass is (normally) green rather than red. The contrastive approach works here also. It might be that explananda in which the fact is the normal case and the foil abnormal are quite rare, but this does not reduce the legitimacy of such questions. At least some of these questions are sensible.

Indeed, this observation suggests one way of characterizing the difference between everyday reasoning and science. The difference is in the explanatory questions asked: in science we also try to explain the normal case, whereas in everyday reasoning we are only interested in explaining the abnormalities. Everyday reasoning takes the normal course of events as granted and only wants to account for a deviation. Usually, we are not able to explain the normal course of events, and this does not bother us. But if something unusual happens, we want to know why. A deviance calls for an explanation. Scientists also explain deviance, but they want to know also about the normal case, and this can be reconstructed as turning around of the usual way of using contrasts. In this sense they are like children.

\section{The question of compatible contrasts}

Peter Lipton (1990) has claimed that not all contrasts are incompatible and a number of authors have accepted this thesis without reservations. (Barnes 1994, Carroll 1997, 1999, Hitchcock 1999, and Risjord 2000.) As this thesis puts my analysis of the contrastive explanandum in danger, let us consider this thesis. Recall the famous example of paresis. Paresis is a form of neurosyphilis, and no one contracts this dreadful disease unless he had latent, untreated syphilis. However, the evolution of the disease is unknown, and only a small percentage of those who have untreated syphilis get paresis. Now we have two persons, Smith, who has had latent syphilis and who now has paresis, and his friend Jones who does not have latent syphilis. We can explain why Smith, rather than his friend, contracted paresis, 
for only he had syphilis. This is something that everybody accepts. However, Lipton notes that the fact that Smith has syphilis is compatible with the possibility that also Jones has syphilis. These two facts are independent of each other and for this reason compatible. It just happens that Jones does not have syphilis. This state of affairs is not in any way related to the curiosities of this particular example. The situation is quite common: we want an explanation for the difference between two apparently similar situations which turned out differently, but which might have turned out similarly. (Lipton 1990: 250.) This is typical in cases of explaining differences, so if Lipton's thesis is right, we might expect that a good many contrasts are compatible.

However, Lipton's thesis is much less radical than it appears. In his 1993 paper he writes:

When we ask questions such as why Smith rather than Jones contracted paresis, our underlying interest often really concerns a contrast about Smith alone. [...] The talk about Jones is a way of getting at a certain type of question about Smith. Thus we see why a contrastive question retains the feeling of incompatibility even when then the explicit contrast is compatible. (Lipton 1993: 46)

Here Lipton admits that the reference to the brother is a surrogate for a counterfactual claim about Smith. We are really interested in his illness, not his friend's health. We are asking why Smith has paresis rather than being like Jones, who does not have paresis? The exclusive alternative in the explanandum is Smith not having paresis, not Jones not having it. It is easy to see that the reference to the brother offers a convenient way of picking the desired contrast about Smith's health, and the explanation is the thing that makes the difference between the two cases. We are contrasting two causal scenarios of Smith's health. The first scenario culminates in Smith having paresis, and the second in Smith being like Jones, that is, without paresis. When subjected to a more careful analysis, the apparently compatible contrasts turn out to be incompatible. Naturally, we could also ask why Jones does not have paresis, when Smith has, but similar considerations will apply. And in some situations we might desire an explanation for both of these contrasts. But in these cases we have two separate explanations, not one explanation with compatible contrasts.

In order to makes sense of this apparent conceptual confusion, we should make a difference between two sorts of explanatory questions. First, there are cases where we are contrasting two alternative outcomes of the same process. Here the contrast is imagined and the fact and its foil are incompatible. In the second case we are contrasting two separate processes with different outcomes. In this case both the fact and the foil are actual. However, in this case there is a difference between the surface structure of the contrastive statement and the 
contrastive structure of the explanatory question. The actual foil is a surrogate for a counterfactual claim about the process that led to the fact to be explained. So the contrast is compatible at the surface level, but incompatible at the deeper level that is the real concern of the theory of explanation.

My claim is that all apparently compatible contrasts turn out to be incompatible when inspected more carefully. I have not seen a single example of compatible contrast that cannot be resolved in this manner. There is good reason for this. The explanation-seeking questions have to be reconstructed in the above manner in order to give them a properly contrastive answer. Otherwise the explanatory counterfactual could not do its job. Looking too closely at the linguistic form of the contrastive statement can lead to a misguided analysis. The basic idea in the contrastive approach to explanation is to look for the implied contrasts, instead of being satisfied with the usual statement of explanandum. The same approach should be used here: one should not be satisfied with just any contrastive statement. Instead, one should look behind linguistic formulations and try to capture the real contrast.

Against this background it is natural to ask what is the point of compatible contrasts if they are so misleading? The reasons are methodological. It is often sensible to try to explain differences between two actual outcomes rather than to explain difference between actual and imagined outcome. First, in the former case one does not have to consider whether the foil is possible outcome of the process. As it has occurred, it is certainly possible. Of course, this presupposes that the cases compared are similar enough. But if they are, there is one potential challenge less for the explanatory question. There is also a second advantage. When explaining the differences between two actual outcomes, one only has to find differences between the two causal histories to find good candidates for the explanans. This much less theoretically burdened process than the one used in explaining the differences between actual and imagined outcomes. Significantly less hangs on one's theoretical understanding of the process, since the whole process of imagining the alternative causal history is cut out. So if the processes are really similar enough, then the explanation of actual differences is much more convincing way to proceed.

\section{Arguments against the contrastive idea}

A very common way to deny the philosophical relevance of contrastive questions in theory of explanation is to claim that not all explanations are contrastive (Ruben 1990: 40; Humphreys 1989: 137). The point implied by this claim is that a philosophical account of explanation need not concern itself with the contingent features of some individual explanations. If only 
some explanations are contrastive, the contrastive approach does not seem suitable for analyzing explanation in general.

Responding to this critique is somewhat tricky. The challenge assumes that the supporters of contrastive approach are making universal statements about all explananda. I have not found any writer on this topic who is committed to this bold claim. For example, Lipton claims to be agnostic about the issue (Lipton 1990: 261). The reason for this is easy to see. Besides the logical problems with proving a universal statement, there is a problem concerning the vague boundaries of what counts as an explanation and what not. People do have conflicting intuitions about the explanatoriness of a good many explanations, and often the putative counterexamples to the contrastive thesis belong to this heterogeneous class of explanations.

I think that a more fruitful approach to understand the contrastive approach would be to interpret it as claiming that all explananda can be analyzed as contrastive. If the contrastive analysis is generally applicable and if in most cases it provides fruitful results, we have an argument for it. I think that the already existing literature on contrastive explanation shows that interesting results can indeed be achieved by the use of the contrastive approach. It might be that in some special cases it does not help much, but this remains to be shown. And, of course, the critics can try to come up with examples where the use of the contrastive idea is a hindrance to the analysis of explanation. I have not seen such examples yet.

The position I am taking can be further clarified by comparing two ways of understanding the contrastive approach to explanation. The first attaches the contrastive idea to a pragmatic theory of explanation. In this approach the contrastive thesis is about what people really have in their minds when they present explanation-seeking questions. The explanation-seeking question is thought to reflect the explainee's epistemic state, and the contrastive suggestion is understood as a way of specifying what the explainee wants to know. I take this to be 'the mainstream approach' in the literature on contrastive explanation. The most famous representative of this approach is Bas van Fraassen (1980).

Dennis Temple has noted a problem with this position. He writes: “... in many cases a speaker who asks 'Why P?' is simply puzzled about P, and without having any particular contrary in mind" (Temple 1988: 147). Temple has a point. Sometimes we do not always have any specific contrast in mind. For example, the explainee can be confused, or she might want answers to many different questions at the same time. Of course, the supporter of the pragmatist approach can reply that his theory is about an ideal or a rational explainee, and not about ordinary people, who are often confused. However, this would be a strange way to defend a pragmatic theory of explanation. 
The defender of the mainstream approach can respond by pointing out that asking for a contrast is a natural and effective way of clarifying or improving the intended explanandum. We can ask the explainee to specify her request by suggesting possible contrasts, such as: "Do you mean why $f$ rather than $c$ or why $f$ rather than $c^{*}$." This is a useful and a very common way to settle the question. (Hesslow 1983: 94.) This is a good point, but I think we do not need the mainstream pragmatic approach to make it.

The alternative interpretation, which I support, takes the contrastive thesis to be a central contribution to a theory about what an explanation can achieve. It is not concerned primarily with the explainee's epistemic states, but with the things that an explanation can do. It asks, given that somebody has provided an explanation, what it explains or which question it answers. It does not make claims about the usual format of the explanation-seeking question, but about the questions for which our explanations could be satisfying answers. We should allow that people could be confused or just simply unclear about their intended explananda. The contrastive thesis should be about what an explanation can explain, not about what kind of questions people have, or can have, in their minds. In this alternative account, it is pragmatic and contextual factors that determine which questions we want to ask or which contrasts we choose, but after fixing the explanatory question the adequacy or inadequacy of the given explanation is a non-pragmatic matter. Consequently, the theory of contrastive explanandum is not a pragmatic theory of explanation in any interesting sense. (Hesslow 1983: 97-98; Woodward 1993: 276.) Of course, it can be naturally extended with pragmatic components, but that is a different matter.

\section{Are contrastive explananda reducible?}

The contrastive approach has also been criticized by claiming that a contrastive explanandum can be reduced to a non-contrastive form. Dennis Temple and later John W. Carroll have suggested that 'Why $f$ rather than $c$ ' is equivalent to 'Why $f$ and not-c' (Temple 1988; Carroll 1997, 1999). Temple claims that a consequence of this reduction of the contrast is that the contrastive approach has no advantage over the traditional 'propositional approach', which sees the explanandum as a (non-contrastive) proposition. Let us call Temple's position 'the conjunctive view'.

Temple makes it sound like the contrastive approach has nothing new to say about the explanandum. This is not true. The traditional explanandum has been the plain ' $f$ ', but now if we accept that the right representation of the explanandum is the conjunction ' $f$ and not-c', we 
have made a substantial point about explanation. Earlier it was not thought that the explanandum is complex in this way.

There are two ways of reading Temple's suggestion. The difference between these readings is whether we accept the following three propositions to be equivalent:

(1) '... explained the fact that $f$ and not-c'

(2) ' $\ldots$ explained the fact that $f$ and explained the fact that not- $c$ '.

(3) '... explained the fact that $f$ rather than $c$ '

The weak reading accepts that (1) and (3) are equivalent, but it does not accept that (2) is equivalent to them. The strong reading accepts that (1), (2), and (3) are all equivalent. The non-conjuctivist position naturally denies all claims of equivalence between these propositions.

Let us first take a look at the strong reading of the conjunctive view. Although neither Temple nor Carroll says it, this reading is really a reductio ad absurdum of the contrastive approach. It claims that contrastive explanations are really conjunctions of two non-contrastive explanations. This would make contrastive explananda quite superficial phenomena. This is ironic: the contrastive suggestion was originally coined to make sense of our ordinary way of explaining 'why $f$ ?'. But now it turns out that we cannot do that because the suggestion presupposes that we already know how to answer this question.

Does this reductive thesis hold water? There are two principal arguments against it. The first argument was advanced by Peter Lipton, who has pointed out that explaining 'Why $f$ rather than $c$ ?' requires less than explaining 'Why $f$ and not- $c$ ?' (Lipton 1990: 252-253). Recall the example of Smith's paresis. No one contracts this dreadful disease unless he has latent, untreated syphilis. However, the evolution of the disease is unknown, and only a small percentage of those who have untreated syphilis get paresis. Now, we can (fully) explain why Smith, rather than his friend Jones, contracted paresis, for only he had syphilis. However, we cannot explain why he, among all syphilitics, got it. Under the assumptions of the example, we don't know why some, but not all, with untreated syphilis contract paresis. The strong reading of the conjunctive view would require that we first explain why Smith contracted paresis, which we cannot do, and then to explain why his brothers did not contract it, which we can do. So, with the conjunctive view we cannot explain something that we intuitively think we can explain. This suggests that the strong reading does not work. We cannot infer (2) '... explained the fact that $f$ and explained the fact that not- $c$ ' from (3) '... explained the fact that $f$ rather than $c^{\prime}$. 
The second argument is by David Ruben. The acceptance of the strong reading requires that there are no limitations on possible contrasts. This can be seen by considering any arbitrary $f$ and not- $c$. Let $f$ be 'snow is white' and let $c$ be 'grass is red'. Suppose that I explain both $f$ and not- $c$. Have I then explained the fact that snow is white rather than grass is red? Clearly something is missing here. We presuppose that there is some sort of relevance between $f$ and $c$ when we contrast them, but the normal truth-functional 'and' does not include any considerations of relevance. (Ruben 1990: 42.) The strong reading does not respect the requirements of relevance of the '... rather than...' locution, which makes it a failed reduction.

What about the weak reading? It does not accept the equivalence between (1) and (2), and so Lipton's and Ruben's arguments cannot refute it. However, these arguments show that the weak reading is of very limited interest. Ruben's argument shows that if we rephrase ' $f$ rather than $c$ ' as ' $f$ and not-c', the 'and' does not work in the normal, truth-functional way. The normal 'and' does not require any relevance, but the ' $f$ rather than $c$ ' requires some relevance between $f$ and $c$. Consequently, the weak reading uses 'and' in non-standard way. There is more than a simple conjunction. This makes Temple's suggestion just an alternative way of indicating the contrast. And this is not big news. As already noted above, the contrast can be expressed by alternative linguistic means.

The fate of Temple's argument teaches us an already familiar lesson: the analysis of contrastive explanation should not focus too closely on linguistic issues. The central focus of interest should be on the cognitive setting, not the linguistic means to express it. Our interest should be in contrastive facts, not in contrastive statements. The danger is that the philosophical analysis regresses to the study of the pragmatics of some locutions in English. And this is not what philosophical analysis should do. After all, explanations are also given in other languages.

\section{References}

Barnes, Eric 1994: 'Why P Rather Than Q? The Curiosities of Fact and Foil', Philosophical Studies 73: 35-53.

Carroll, John W. 1997: 'Lipton on compatible contrasts', Analysis 57: 170-178.

Carroll, John W. 1999: 'The Two Dams and That Damned Paresis', The British Journal for the Philosophy of Science 50: 65-81.

Cummins, Robert 1983: The Nature of Psychological Explanation. MIT Press. Cambridge.

Garfinkel, Alan 1981: Forms of Explanation. Yale University Press. New Haven.

Hansson, Bengt 1975: 'Explanations - Of What?', unpublished manuscript.

Hart, H. L. A. \& Honoré, A. M. 1959: Causation in the Law. Claredon Press. Oxford.

Hempel, Carl 1965: Aspects of Scientific Explanation. The Free Press. New York. 
Hesslow, Germund 1983: 'Explaining differences and weighting causes', Theoria 49: 87-111.

Hilton, Denis J. 1995: 'Logic and language in causal explanation', in Sperber, Premack \& Premack (eds.): Causal Cognition. Oxford University Press, Oxford: 495-525.

Hintikka, Jaakko \& Halonen, Ilpo 1995: 'Semantics and Pragmatics for WhyQuestions', The Journal of Philosophy 92: 636-657.

Hitchcock, Christopher C. 1996: 'The Role of Contrast in Causal and Explanatory Claims', Synthese 107: 395-419.

Hitchcock, Christopher C. 1999: 'Contrastive Explanation and the Demons of Determinism', The British Journal for the Philosophy of Science 50: 585612.

Hållsten, Henrik 2001: Explanation and Deduction. A Defence of Deductive Chauvinism. Acta Universitatis Stockholmiensis. Stockholm Studies in Philosophy 21. Almqvist \& Wiksell. Stockholm.

Humphreys, Paul 1989: The Chances of Explanation. Princeton University Press. Princeton.

Koura, Antti 1988: ‘An Approach to Why-Questions', Synthese 74: 191-206.

Lewis, David 1986: Philosophical Papers vol II. Oxford University Press. Oxford.

Lipton, Peter 1990: 'Contrastive Explanations', in Knowles (ed.): Explanation and its Limits. Cambridge University Press. Cambridge: 247-266.

Lipton, Peter 1991: Inference to the Best Explanation. Routledge. London.

Lipton, Peter 1993: 'Making a Difference', Philosophica 51: 39-54.

Markwick, P. 1999: 'Interrogatives and Contrasts in Explanation Theory', Philosophical Studies 96: 183-204.

Railton, Peter 1978: 'A Deductive-Nomological Model of Probabilistic Explanation', Philosophy of Science 45: 206-226.

Railton, Peter 1981: 'Probability, Explanation, and Information', Synthese 48: 233-256.

Risjord, Mark W. 2000: Woodcutters and Witchcraft. SUNY. Albany.

Ruben, David-Hillel 1990: Explaining Explanation. Routledge. London.

Scriven, Michael 1959: 'Truisms as the Grounds for Historical Explanations', in Gardiner (ed.): Theories of History. The Free Press. New York: 443-475.

Sintonen, Matti 1984: The Pragmatics of Scientific Explanation. Acta Philosophica Fennica 37. Societas Philosophica Fennica. Helsinki.

Sober, Elliot 1994: 'Explanatory Presupposition', in From a Biological Point of View. Cambridge University Press. Cambridge, 175-183.

Temple, Dennis 1988: 'The Contrast Theory of Why-Questions', Philosophy of Science 55: 141-151.

Toulmin, Stephen 1961: Foresight and Understanding. Hutchinson. London.

Tuomela, Raimo 1980: 'Explaining Explaining', Erkenntnis 15: 211-243.

van Fraassen, Bas 1980: Scientific Image. Oxford University Press. Oxford.

Woodward, James 1993: 'A Theory of Singular Causal Explanation', in Ruben (ed.): Explanation. Oxford University Press. Oxford: 246-274.

Woodward, James 2003: Making Things Happen. A Theory of Causal Explanation. Oxford University Press. Oxford.

Ylikoski, Petri 2001: Understanding Interests and Causal Explanation. Ph.D. thesis. May 2001. [http://ethesis.helsinki.fi/julkaisut/val/kayta/vk/ylikoski/] 